PROCEEDINGS OF THE

AMERICAN MATHEMATICAL SOCIETY

Volume 137, Number 1, January 2009, Pages 219-225

S 0002-9939(08)09486-0

Article electronically published on May 22, 2008

\title{
THE SCHUR PROPERTY ON PROJECTIVE AND INJECTIVE TENSOR PRODUCTS
}

\author{
GERALDO BOTELHO AND PILAR RUEDA
}

(Communicated by N. Tomczak-Jaegermann)

\begin{abstract}
The problem of whether the Schur property is passed from a Banach space to its (symmetric) projective $n$-fold tensor product is reformulated in the language of polynomial ideals. As a result, a very closely related question is solved in the negative. It is also proved that the injective tensor product of infrabarrelled locally convex spaces with the Schur property has the Schur property as well.
\end{abstract}

\section{INTRODUCTION}

It is an open problem, which seems to be a very difficult one, whether the Schur property (weakly convergent sequences are convergent) is passed from a Banach space to its $n$-fold (symmetric) projective tensor product. In this paper we reformulate this question in terms of polynomial ideals, more precisely in terms of spaces of completely continuous homogeneous polynomials between Banach spaces. Doing so we solve in the negative a very closely related problem, so an eventual negative answer to the original problem will not be a surprise. A well-known result due to Lust [14] asserts that the Schur property is stable under the formation of injective tensor products of Banach spaces. In the final section we extend this result to a rather large class of locally convex spaces, namely infrabarrelled spaces.

\section{Preliminaries}

Given locally convex spaces $E$ and $F, \mathcal{L}(E ; F)$ denotes the space of all continuous linear operators from $E$ into $F$ endowed with the topology of uniform convergence on bounded subsets of $E$ (if $F$ is the scalar field we simply write $F^{*}$ ). If $\mathcal{B}$ denotes the set of all bounded subsets of $E$ and $\mathcal{V}$ is a base of absolutely convex 0 -neighborhoods in $F$, then the family

$$
\{\{u \in \mathcal{L}(E ; F): u(A) \subset B\}, A \in \mathcal{B} \text { and } B \in \mathcal{V}\}
$$

forms a base of 0 -neighborhoods in $\mathcal{L}(E ; F)$. By $E \hat{\otimes}_{\varepsilon} F$ and $E \hat{\otimes}_{\pi} F$ we mean respectively the completed injective and projective tensor products of $E$ and $F$. The completed $n$-fold injective and projective tensor products of $E$ are denoted by $\hat{\otimes}_{\varepsilon}^{n} E$

Received by the editors September 24, 2007, and, in revised form, December 27, 2007.

2000 Mathematics Subject Classification. Primary 46G20; Secondary 46A04, 46A32.

The first author was supported by CNPq Project 202162/2006-0.

The second author was supported by MEC and FEDER Project MTM2005-08210.

(C) 2008 American Mathematical Society Reverts to public domain 28 years from publication 
and $\hat{\otimes}_{\pi}^{n} E$ respectively. The notation $\hat{\otimes}_{\pi}^{n, s} E$ stands for the completed projective $n$-fold symmetric tensor product of $E$.

Given locally convex spaces $E$ and $F$ and a positive integer $n, \mathcal{P}\left({ }^{n} E ; F\right)$ and $\mathcal{L}\left({ }^{n} E ; F\right)$ denote the locally convex spaces of all continuous $n$-homogeneous polynomials from $E$ into $F$ and continuous $n$-linear mappings from $E^{n}$ to $F$, respectively, endowed with the usual strong topology. If $F$ is the scalar field we simply write $\mathcal{P}\left({ }^{n} E\right)$ and $\mathcal{L}\left({ }^{n} E\right)$. Let $P_{L}$ denote the linearization of the polynomial $P \in \mathcal{P}\left({ }^{n} E ; F\right)$, that is,

$$
P_{L} \in \mathcal{L}\left(\hat{\otimes}_{\pi}^{n, s} E ; F\right) \text { and } P(x)=P_{L}(x \otimes \cdots \otimes x) \text { for every } x \in E .
$$

The correspondence $P \in \mathcal{P}\left({ }^{n} E ; F\right) \mapsto P_{L} \in \mathcal{L}\left(\hat{\otimes}_{\pi}^{n, s} E ; F\right)$ establishes an isomorphism onto. The linearization of homogeneous polynomials is due to Ryan [17.

Given an open subset $U$ of a complex locally convex space $E, \mathcal{H}(U ; F)$ represents the space of all holomorphic mappings from $U$ into $F$ (again $\mathcal{H}(U ; \mathbb{C})=\mathcal{H}(U)$ ). A bounded subset $A$ of an open set $U \subseteq E$ is said to be $U$-bounded if there is a 0-neighborhood $V$ such that $A+V \subseteq U$. A function $f \in \mathcal{H}(U ; F)$ is said to be of bounded type, in symbols $f \in \mathcal{H}_{b}(U ; F)$, if $f$ is bounded on $U$-bounded sets. By $\mathcal{H}_{w u}(U ; F)$ we mean the space of all holomorphic mappings from $U$ to $F$ that are weakly uniformly continuous on $U$-bounded sets. Both $\mathcal{H}_{b}(U ; F)$ and $\mathcal{H}_{w u}(U ; F)$ are endowed with the topology of uniform convergence on $U$-bounded sets.

For the general theory of homogeneous polynomials and holomorphic functions we refer to Dineen $[9]$.

\section{Projective tensor Products}

Let $E$ be a Banach space and $n$ be a positive integer. In this section we address the open question (see [11, Remark 6])

$$
\hat{\otimes}_{\pi}^{n, s} E \text { has the Schur property } \stackrel{? ?}{\Longrightarrow} \hat{\otimes}_{\pi}^{n+1, s} E \text { has the Schur property, }
$$

which is likely to be very difficult. Our aim is to reformulate this problem using the language of polynomial ideals. In this new approach we are able to prove that a very closely related problem has a negative answer, a fact we believe is symptomatic. In this section all spaces are supposed to be Banach.

Completely continuous linear operators (weakly null sequences are sent onto norm null sequences) are related to the Schur property by the trivial fact that a Banach space $E$ has the Schur property if and only if the identity operator on $E$ is completely continuous. So, having in mind that the spaces $\mathcal{L}\left(\hat{\otimes}_{\pi}^{n, s} E ; F\right)$ and $\mathcal{P}\left({ }^{n} E ; F\right)$ are isomorphic, in order to connect the Schur property on a Banach space $E$ with the Schur property on $\hat{\otimes}_{\pi}^{n, s} E$ it is natural to consider a polynomial generalization of the ideal of completely continuous operators. Rather than the widely studied class of weakly sequentially continuous polynomials, another polynomial generalization of completely continuous operators shall be useful:

Definition 3.1 (Composition ideals; see [6]). By $C C$ we denote the ideal of completely continuous linear operators and by $C C \circ \mathcal{P}$ the corresponding composition ideal of polynomials. Recall that a polynomial $P \in \mathcal{P}\left({ }^{n} E ; F\right)$ belongs to $C C \circ \mathcal{P}$, in symbols $P \in C C \circ \mathcal{P}\left({ }^{n} E ; F\right)$, if there exist a Banach space $G$, an operator $u \in C C(G ; F)$ and $Q \in \mathcal{P}\left({ }^{n} E ; G\right)$ such that $P=u \circ Q$. 
Example 3.2. It is clear that $\mathcal{P}\left({ }^{n} E\right)=C C \circ \mathcal{P}\left({ }^{n} E\right)$ for every $n$ and any $E$. Our aim in this example is to show that this coincidence is no longer true in general for vector-valued polynomials. Let $p>2$ and choose a continuous linear functional $\varphi \neq 0$ on $\ell_{p}$. Consider the polynomial

$$
P: \ell_{p} \longrightarrow \ell_{p} ; P(x)=\varphi(x) x .
$$

Suppose that $P \in C C \circ \mathcal{P}\left({ }^{2} \ell_{p} ; \ell_{p}\right)$. By [6, Proposition 2.2] we have that the linear operator $P_{L}: \ell_{p} \hat{\otimes}_{\pi}^{s} \ell_{p} \longrightarrow \ell_{p}$ is completely continuous. By [8, Example 4.1] we know that $\mathcal{P}\left({ }^{2} \ell_{p}\right)=\left(\ell_{p} \hat{\otimes}_{\pi}^{s} \ell_{p}\right)^{*}$ is reflexive, so $\ell_{p} \hat{\otimes}_{\pi}^{s} \ell_{p}$ is reflexive as well. Completely continuous linear operators on reflexive spaces are compact [12, Proposition 17.1.10], so $P_{L}$ is compact. Defining $\delta_{2}: \ell_{p} \longrightarrow \ell_{p} \hat{\otimes}_{\pi}^{s} \ell_{p}$ by $\delta_{2}(x)=x \otimes x$, the factorization $P=P_{L} \circ \delta_{2}$ yields that $P$ is a compact polynomial. This is absurd because $\ell_{p}$ is infinite-dimensional (see [5, p. 461]), so $P \notin C C \circ \mathcal{P}\left({ }^{2} \ell_{p} ; \ell_{p}\right)$. Of course this construction works for every infinite-dimensional Banach space $E$ such that $\mathcal{P}\left({ }^{2} E\right)$ is reflexive, for instance for Tsirelson's original space $T^{*}$ (Alencar, Aron and Dineen [1] proved that $\mathcal{P}\left({ }^{n} T^{*}\right)$ is reflexive for every $\left.n\right)$.

The coincidence $\mathcal{P}=C C \circ \mathcal{P}$ for vector-valued polynomials on $E$ makes the connection with the original problem concerning the Schur property on $\hat{\otimes}_{\pi}^{n, s} E$ :

Proposition 3.3. Let $E$ be a Banach space and $n$ be a positive integer. Then $\hat{\otimes}_{\pi}^{n, s} E$ has the Schur property if and only if $\mathcal{P}\left({ }^{n} E ; F\right)=C C \circ \mathcal{P}\left({ }^{n} E ; F\right)$ for every Banach space $F$.

Proof. Suppose that $\hat{\otimes}_{\pi}^{n, s} E$ has the Schur property. Let $P \in \mathcal{P}\left({ }^{n} E ; F\right), P=P_{L} \circ \delta_{n}$, where $\delta_{n}: E \longrightarrow \hat{\otimes}_{\pi}^{n, s} E$ is the canonical $n$-homogeneous polynomial defined by $\delta_{n}(x)=x \otimes \cdots \otimes x . P_{L}$ is completely continuous as $\hat{\otimes}_{\pi}^{n, s} E$ has the Schur property, so $P \in C C \circ \mathcal{P}\left({ }^{n} E ; F\right)$. Conversely, as $\delta_{n}$ is continuous, $\delta_{n} \in C C \circ \mathcal{P}\left({ }^{n} E ; \hat{\otimes}_{\pi}^{n, s} E\right)$ by assumption. By [6, Proposition 2.2] it follows that $\left(\delta_{n}\right)_{L} \in C C\left(\hat{\otimes}_{\pi}^{n, s} E ; \hat{\otimes}_{\pi}^{n, s} E\right)$. But $\left(\delta_{n}\right)_{L}$ is the identity operator, so $\hat{\otimes}_{\pi}^{n, s} E$ has the Schur property.

Example 3.4. As $\hat{\otimes}_{\pi}^{n, s} \ell_{1}$ is topologically isomorphic to $\hat{\otimes}_{\pi}^{n} \ell_{1}$ [3, Lemma 5.2] and the latter space is topologically isomorphic to $\ell_{1}$, which has the Schur property, $\ell_{1}$ fulfills the equivalent conditions of Proposition 3.3 for every $n$. Bourgain and Pisier [7] constructed an infinite-dimensional Banach space $B P$ with the Schur property such that $B P \hat{\otimes}_{\pi} B P=B P \hat{\otimes}_{\varepsilon} B P$. By Lust's result [14 it follows that $B P \hat{\otimes}_{\pi} B P$ has the Schur property, so Proposition 3.3 gives that $\mathcal{P}\left({ }^{2} B P ; F\right)=C C \circ \mathcal{P}\left({ }^{2} B P ; F\right)$ for every Banach space $F$. We do not know whether $\hat{\otimes}_{\pi}^{n} B P$ has the Schur property for $n>2$. John [13] proved that if $n \geq 3$ and the injective and projective norms are equivalent on $\otimes_{n} E$, then $E$ is finite dimensional. So, for $n \geq 3, \hat{\otimes}_{\pi}^{n} B P$ and $\hat{\otimes}_{\varepsilon}^{n} B P$ no longer coincide.

Remark 3.5. It is well known (see [15, 17]) that a polynomial $P \in \mathcal{P}\left({ }^{n} E ; F\right)$ is compact (resp. weakly compact) if and only if $P$ admits a factorization $P=u \circ Q$ where $G$ is a Banach space, $Q \in \mathcal{P}\left({ }^{n} E ; G\right)$ and $u: G \longrightarrow F$ is a compact (resp. weakly compact) linear operator. So, the condition $\mathcal{P}\left({ }^{n} E ; F\right)=C C \circ \mathcal{P}\left({ }^{n} E ; F\right)$ in Proposition 3.3 addresses the validity of a similar factorization of polynomials through completely continuous linear operators.

From Proposition 3.3 it is easy to see that if $\mathcal{P}\left({ }^{n} E ; F\right)=C C \circ \mathcal{P}\left({ }^{n} E ; F\right)$ for every $F$, then $\mathcal{P}\left({ }^{m} E ; F\right)=C C \circ \mathcal{P}\left({ }^{m} E ; F\right)$ for every $F$ and any $1 \leq m \leq n$. Actually much more is true: 
Proposition 3.6. Let $E$ and $F$ be Banach spaces and $n \in \mathbb{N}$. If $\mathcal{P}\left({ }^{n} E ; F\right)=$ $C C \circ \mathcal{P}\left({ }^{n} E ; F\right)$, then $\mathcal{P}\left({ }^{m} E ; F\right)=C C \circ \mathcal{P}\left({ }^{m} E ; F\right)$ for every $1 \leq m \leq n$.

Proof. Given $P \in \mathcal{P}\left({ }^{m} E ; F\right), P_{L} \in \mathcal{L}\left(\hat{\otimes}_{\pi}^{m, s} E ; F\right)$. By [4, Corollary 4] there is a complemented subspace $E_{n}^{m}$ of $\hat{\otimes}_{\pi}^{n, s} E$ and an isomorphism $u: E_{n}^{m} \longrightarrow \hat{\otimes}_{\pi}^{m, s} E$. Since $E_{n}^{m}$ is complemented in $\hat{\otimes}_{\pi}^{n, s} E, P_{L} \circ u$ admits an extension $\widetilde{P_{L} \circ u}$ to $\hat{\otimes}_{\pi}^{n, s} E$ so that the diagram

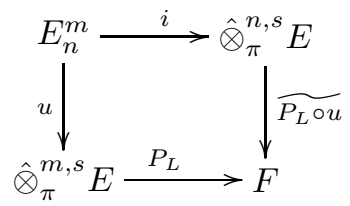

where $i$ is the formal inclusion, is commutative. But $\mathcal{L}\left(\hat{\otimes}_{\pi}^{n, s} E ; F\right)$ is isomorphic to $\mathcal{P}\left({ }^{n} E ; F\right)$ via the linearization operator, so there exists $Q \in \mathcal{P}\left({ }^{n} E ; F\right)$ such that $Q_{L}=\widetilde{P_{L} \circ u} . Q \in C C \circ \mathcal{P}\left({ }^{n} E ; F\right)$ by assumption, so $Q_{L}=\widetilde{P_{L} \circ u}$ is completely continuous by [6, Proposition 2.2]. It follows that $P_{L}=\left(\widetilde{P_{L} \circ u}\right) \circ i \circ u^{-1}$ is completely continuous by the ideal property; hence $P \in C C \circ \mathcal{P}\left({ }^{m} E ; F\right)$.

From Proposition 3.3 , given a Banach space $E$, problem $\left(^{*}\right)$ is equivalent to

$$
\mathcal{P}\left({ }^{n} E ; F\right)=C C \circ \mathcal{P}\left({ }^{n} E ; F\right), \forall F \stackrel{? ?}{\Longrightarrow} \mathcal{P}\left({ }^{n+1} E ; F\right)=C C \circ \mathcal{P}\left({ }^{n+1} E ; F\right), \forall F .
$$

We finish this section by showing that the closely related implication

$$
\mathcal{P}\left({ }^{n} E ; F\right)=C C \circ \mathcal{P}\left({ }^{n} E ; F\right) \Longrightarrow \mathcal{P}\left({ }^{n+1} E ; F\right)=C C \circ \mathcal{P}\left({ }^{n+1} E ; F\right),
$$

which happens to be an 'increasing version' of Proposition 3.6, does not hold true in general, so an eventual negative answer to question $\left(^{*}\right)$ will not be a surprise.

Proposition 3.7. Let $n \in \mathbb{N}$ and $p, q \in(1,+\infty)$ be such that $n q<p \leq(n+1) q$ and $p>n+1$. Then

$$
\mathcal{P}\left({ }^{n} \ell_{p} ; \ell_{q}\right)=C C \circ \mathcal{P}\left({ }^{n} \ell_{p} ; \ell_{q}\right) \text { and } \mathcal{P}\left({ }^{n+1} \ell_{p} ; \ell_{q}\right) \neq C C \circ \mathcal{P}\left({ }^{n+1} \ell_{p} ; \ell_{q}\right) \text {. }
$$

In particular,

$$
\mathcal{P}\left({ }^{2} \ell_{5} ; \ell_{2}\right)=C C \circ \mathcal{P}\left({ }^{2} \ell_{5} ; \ell_{2}\right) \text { and } \mathcal{P}\left({ }^{3} \ell_{5} ; \ell_{2}\right) \neq C C \circ \mathcal{P}\left({ }^{3} \ell_{5} ; \ell_{2}\right) .
$$

Proof. Let $P \in \mathcal{P}\left({ }^{n} \ell_{p} ; \ell_{q}\right)$ be given. As $n q<p$, 2, Theorem 4.2] gives that $P$ is compact. By [17, Lemma 4.1] it follows that the linear operator $P_{L}: \hat{\otimes}_{\pi}^{n, s} \ell_{p} \longrightarrow F$ is compact, hence completely continuous (see the proof of [12, Proposition 17.1.10]). The factorization $P=P_{L} \circ \delta_{n}$ shows that $P \in C C \circ \mathcal{P}\left({ }^{n} \ell_{p} ; \ell_{q}\right)$.

The $(n+1)$-homogeneous polynomial $P \in \mathcal{P}\left({ }^{n+1} \ell_{p} ; \ell_{q}\right)$ given by $P\left(\left(\alpha_{j}\right)_{j=1}^{\infty}\right)=$ $\left(\alpha_{j}^{n+1}\right)_{j=1}^{\infty}$ is well defined and continuous because $p \leq(n+1) q$. Suppose that $P \in C C \circ \mathcal{P}\left({ }^{n+1} \ell_{p} ; \ell_{q}\right)$. Reasoning as in Example $3.2\left(\mathcal{P}\left({ }^{n+1} \ell_{p}\right)\right.$ is reflexive by [8, Example 4.1] because $p>n+1)$, it follows that $P$ is compact. This is absurd because the sequence $\left(e_{j}\right)_{j=1}^{\infty}$ is bounded in $\ell_{p}$ and $\left(P\left(e_{j}\right)\right)_{j=1}^{\infty}=\left(e_{j}\right)_{j=1}^{\infty}$ has no convergent subsequence in $\ell_{q}$, which proves that $P \notin C C \circ \mathcal{P}\left({ }^{n+1} \ell_{p} ; \ell_{q}\right)$. 


\section{INJECTIVE TENSOR PRODUCTS}

As mentioned earlier, Lust [14] proved that the Schur property is stable under the formation of injective tensor products of Banach spaces. By adapting a proof due to Ryan [18] of Lust's result, we generalize it to a quite large class of locally convex spaces. Recall that a locally convex space $E$ is infrabarrelled if the inclusion from $E$ into its strong bidual $E^{* *}$ is a topological isomorphism onto its range. Barrelled spaces and metrizable spaces are infrabarrelled [16, Propositions V.9 and IV.2].

Proposition 4.1. Let $E$ and $F$ be locally convex spaces with $E$ infrabarrelled. If $E$ and $F$ both have the Schur property, then the (closed) subspace $\mathcal{L}_{w^{*}}\left(E^{*} ; F\right)$ of $\mathcal{L}\left(E^{*} ; F\right)$ of all weak ${ }^{*}$-to-weak continuous operators has the Schur property. In particular, E $\hat{\otimes}_{\varepsilon} F$ has the Schur property.

Proof. Let $\left(u_{n}\right)$ be a sequence in $\mathcal{L}_{w^{*}}\left(E^{*} ; F\right)$ not converging to 0 . Then there is a strong bounded set $A$ in $E^{*}$, a 0 -neighborhood $B$ in $F$ and a strictly increasing sequence of positive integers $\left(n_{k}\right)_{k}$ such that each $u_{n_{k}}$ does not belong to the set $\left\{u \in \mathcal{L}_{w^{*}}\left(E^{*} ; F\right): u(A) \subset B\right\}$. Then, for every $k$ there is $a_{k} \in A$ such that $u_{n_{k}}\left(a_{k}\right) \notin B$. This means that $\left(u_{n_{k}}\left(a_{k}\right)\right)_{k}$ is not a null sequence in $F$, hence not weakly null because $F$ has the Schur property. Then there are a linear functional $y^{*} \in F^{*}, \delta>0$ and a strictly increasing sequence of positive integers $\left(k_{l}\right)_{l}$ such that $\left|y^{*}\left(u_{n_{k_{l}}}\left(a_{k_{l}}\right)\right)\right|>\delta$ for all $l$. As $y^{*} \circ u_{n_{k_{l}}}=u_{n_{k_{l}}}^{t}\left(y^{*}\right) \in E$ (an operator $v: E^{*} \longrightarrow F$ belongs to $\mathcal{L}_{w^{*}}\left(E^{*} ; F\right)$ if and only if its transpose $v^{t}$ is $E$-valued) and $E$ is infrabarrelled, the above inequality shows that $\left(u_{n_{k_{l}}}^{t}\left(y^{*}\right)\right)_{l}$ is not a null sequence in $E$. But $E$ has the Schur property, so the sequence $\left(u_{n_{k_{l}}}^{t}\left(y^{*}\right)\right)_{l}$ fails to be weakly null in $E$. Therefore the sequence $\left(x^{*}\left(u_{n_{k_{l}}}^{t}\left(y^{*}\right)\right)\right)_{l}$ does not converge to 0 for some $x^{*} \in E^{*}$. The map $\phi$ given by $\phi(u)=x^{*}\left(u^{t}\left(y^{*}\right)\right)$ is a continuous linear operator on $\mathcal{L}_{w^{*}}\left(E^{*} ; F\right)$, and we have seen that $\left(\phi\left(u_{n_{k_{l}}}\right)\right)_{l}$ is not a null sequence. This shows that $\left(u_{n_{k_{l}}}\right)_{l}$, hence $\left(u_{n}\right)$, is not weakly null, proving that $\mathcal{L}_{w^{*}}\left(E^{*} ; F\right)$ has the Schur property. The second assertion follows as $E \hat{\otimes}_{\varepsilon} F$ may be canonically embedded in $\mathcal{L}_{w^{*}}\left(E^{*} ; F\right)$.

The proof above can also be used to generalize [18, Theorem 3.3(b)] and [10, Corollary 4.11] to the locally convex setting. A sequence $\left(E_{n}\right)_{n}$ of subspaces of a locally convex space $E$ is said to be a Schauder decomposition of $E$ if any $x \in E$ can be written in a unique way as $x=\sum_{n=1}^{\infty} x_{n}$ with $x_{n} \in E_{n}$ for every $n$ and the projection $\sum_{n=1}^{\infty} x_{n} \mapsto \sum_{n=1}^{m} x_{n}$ is continuous for every $m \in \mathbb{N}$. Let $\mathcal{S}$ denote the set of all scalar sequences $\left(\alpha_{n}\right)_{n}$ such that $\lim \sup _{n}\left|\alpha_{n}\right|^{1 / n} \leq 1$. A Schauder decomposition $\left(E_{n}\right)_{n}$ of $E$ is $\mathcal{S}$-absolute (see [9]) if for all $\left(\alpha_{n}\right)_{n} \in \mathcal{S}$ and all $x=\sum_{n=1}^{\infty} x_{n} \in E$, the series $\sum_{n=1}^{\infty} \alpha_{n} x_{n}$ converges in $E$ and for each continuous seminorm $p$ on $E$ and each $\alpha=\left(\alpha_{n}\right)_{n} \in \mathcal{S}$, the expression $p_{\alpha}\left(\sum_{n=1}^{\infty} x_{n}\right)=\sum_{n=1}^{\infty}\left|\alpha_{n}\right| p\left(x_{n}\right)$ defines a continuous seminorm on $E$.

Lemma 4.2. A locally convex space $E$ with an $\mathcal{S}$-absolute Schauder decomposition $\left(E_{n}\right)_{n}$ has the Schur property if and only if each $E_{n}$ has the Schur property.

Proof. Assume that $E_{n}$ has the Schur property for every $n$ and let us prove that $E$ has the Schur property. To do so, let $\left(x_{m}\right)$ be a weakly null sequence in $E$. Each $x_{m}$ can be written as $x_{m}=\sum_{n=1}^{\infty} x_{n}^{m}, x_{n}^{m} \in E_{n}$. Since $\left(E_{n}\right)_{n}$ is a Schauder decomposition of $E$, the continuity of the projections yields that $\left(x_{n}^{m}\right)_{m}$ is a weakly null sequence in $E_{n}$ for every $n$. Then $\left(x_{n}^{m}\right)_{m}$ converges to 0 in $E_{n}$ for every $n$. 
Using that the decomposition is $\mathcal{S}$-absolute, the sequence $\left(x_{m}\right)_{m}$ converges to 0 in $E$ by [9, Proposition 3.34]. The converse is clear.

Proposition 4.3. Let $E$ and $F$ be complex locally convex spaces. The following assertions are equivalent:

(1) $E^{*}$ and $F$ have the Schur property.

(2) $\mathcal{L}(E ; F)$ has the Schur property.

(3) $\mathcal{L}\left({ }^{n} E ; F\right)$ has the Schur property for every $n$.

(4) $\mathcal{P}\left({ }^{n} E ; F\right)$ has the Schur property for every $n$.

(5) $\mathcal{H}_{b}(U ; F)$ has the Schur property for every balanced open subset $U \subset E$.

(6) $\mathcal{H}_{w u}(U ; F)$ has the Schur property for every balanced open subset $U \subset E$.

Proof. Virtually the same proof of Proposition 4.1 gives $(1) \Longrightarrow(2)$. Observe that in this case we do not come from $E^{* *}$ back to $E$, so there is no need for $E$ to be infrabarrelled. Assuming (2), it is clear that $E^{*}$ and $F$ have the Schur property. Since $\mathcal{L}\left({ }^{n} E ; F\right)$ and $\mathcal{L}\left(E ; \mathcal{L}\left({ }^{n-1} E ; F\right)\right)$ are isomorphic for every $n \geq 2$, induction on $n$ combined with $(1) \Longrightarrow(2)$ gives (3). An adaptation of the proof of [9, Proposition 3.36] shows that the spaces $\left(\mathcal{P}\left({ }^{n} E ; F\right)\right)_{n}$ form an $\mathcal{S}$-absolute decomposition of $\mathcal{H}_{b}(U ; F)$, so (4) $\Longrightarrow(5)$ follows from Lemma 4.2, The proof is complete as $(2) \Longrightarrow(1),(3) \Longrightarrow(4)$ and $(5) \Longrightarrow(6) \Longrightarrow(1)$ are easy.

\section{ACKNOWLEDGEMENTS}

This paper was written while the first author was a CNPq Postdoctoral Fellow in the Departamento de Análisis Matemático at Universidad de Valencia. He thanks Pilar Rueda and the members of the department for their kind hospitality. The authors thank J. Bonet, C. Boyd, and the anonymous referee for their helpful suggestions.

\section{REFERENCES}

[1] R. Alencar, R. Aron and S. Dineen. A reflexive space of holomorphic functions in infinitely many variables, Proc. Amer. Math. Soc. 90 (1984), no. 3, 407-411. MR728358 (85b:46050)

[2] R. Alencar and K. Floret. Weak-strong continuity of multilinear mappings and the PetczyńskiPitt theorem, J. Math. Anal. Appl. 206 (1997), 532-546. MR1433955 (98h:46045)

[3] A. Arias and J. Farmer. On the structure of tensor products of $\ell_{p}$-spaces, Pacific J. Math. 175 (1996), no. 1, 13-37. MR1419470 (98d:46015)

[4] F. Blasco. Complementation in spaces of symmetric tensor products and polynomials, Studia Math. 123 (1997), no. 2, 165-173. MR1439028 (98d:46043)

[5] G. Botelho. Weakly compact and absolutely summing polynomials, J. Math. Anal. Appl. 265 (2002), no. 2, 458-462. MR 1876152 (2002j:46052)

[6] G. Botelho, D. Pellegrino and P. Rueda. On composition ideals of multilinear mappings and homogeneous polynomials, Publ. Res. Inst. Math. Sci. 43 (2007), no. 4, 1139-1155. MR2389796

[7] J. Bourgain and G. Pisier. A construction of $\mathcal{L}_{\infty}$-spaces and related Banach spaces, Bol. Soc. Bras. Mat. 14 (1983), no. 2, 109-123. MR.756904 (86b:46021)

[8] V. Dimant and I. Zalduendo. Bases in spaces of multilinear forms over Banach spaces, J. Math. Anal. Appl. 200 (1996), 548-566. MR1393101 (97k:46017)

[9] S. Dineen. Complex Analysis on Infinite-Dimensional Spaces, Springer-Verlag London, Ltd., London, 1999. MR.1705327 (2001a:46043)

[10] M. González and J. Gutiérrez. Gantmacher type theorems for holomorphic mappings, Math. Nachr. 186 (1997), 131-145. MR.1461217(99b:46068)

[11] M. González and J. Gutiérrez. The Dunford-Pettis property on tensor products, Math. Proc. Cambridge Philos. Soc. 131 (2001), no. 1, 185-192. MR1833082 (2002d:46014)

[12] H. Jarchow. Locally Convex Spaces, B. G. Teubner, Stuttgart, 1981. MR632257 (83h:46008) 
[13] K. John. Tensor product of several spaces and nuclearity, Math. Ann. 269 (1984), no. 3, 333-356. MR761310 (86b:46119)

[14] F. Lust. Produits tensoriels injectifs d'espaces de Sidon, Colloq. Math. 32 (1975), 285-289. MR0390794 (52:11617)

[15] A. Pełczyński. On weakly compact polynomial operators on B-spaces with Dunford-Pettis property, Bull. Acad. Polon. Sci. Sér. Sci. Math. Astronom. Phys. 11 (1963), 371-378. MR0161160 (28:4369)

[16] A. P. Robertson and W. Robertson. Topological Vector Spaces, Cambridge University Press, London-New York, 1973. MR0350361 (50:2854)

[17] R. Ryan. Applications of topological tensor products to infinite dimensional holomorphy, Thesis, Trinity College, 1980.

[18] R. Ryan. The Dunford-Pettis property and projective tensor products, Bull. Polish Acad. Sci. Math. 35 (1987), no. 11-12, 785-792. MR961717 (89k:46019)

Faculdade de Matemática, Universidade Federal de Uberlândia, 38.400-902, UBERLÂNDIA, BRAZIL

E-mail address: botelho@ufu.br

Departamento de Análisis Matemático, Universidad de Valencia, 46.100 Burjasot, VALENCIA, SPAin

E-mail address: pilar.rueda@uv.es 